\title{
Family Dependence in 331 Models
}

\author{
R. Martínez and F. Ochoa \\ Universidad Nacional de Colombia, Ciudad Universitaria, \\ Carrera 30, No. 45-03, Edificio 405 Oficina 218, Bogota, Colombia
}

Received on 16 October, 2006

\begin{abstract}
Using experimental results at the Z-pole, and considering the ansatz of Matsuda as an specific texture for the quark mass matrices, we perform a $\chi^{2}$ fit at $95 \%$ CL to obtain family-dependent bounds to $Z^{\prime}$ mass and Z-Z' mixing angle in the framework of the main versions of 331 models. The allowed regions depend on the assignment of the physical quark families into different representations that cancel anomalies. Allowed regions on other possible 331 models are also obtained.
\end{abstract}

Keywords: Standart model; Gauge bosons

\section{INTRODUCTION}

In most of extensions of the standard model (SM), new massive and neutral gauge bosons, called $Z^{\prime}$, are predicted. The presence of this boson is sensitive to experimental observations at low and high energies, and will be of great interest in the next generation of colliders (LHC, ILC, TESLA) [1]. In particular, it is possible to study some phenomenological features associated to $Z^{\prime}$ through models with gauge symmetry $S U(3)_{c} \otimes S U(3)_{L} \otimes U(1)_{X}$, also called 331 models. These models arise as an interesting alternative to explain the origin of generations [2-4], where the three families are required in order to cancel chiral anomalies. The electric charge is defined as a linear combination of the diagonal generators of the group

$$
Q=T_{3}+\beta T_{8}+X I
$$

where $\beta$ allow classify the different 331 models. The two main versions corresponds to $\beta=-\sqrt{3}$ [2] and $\beta=-\frac{1}{\sqrt{3}}$ [3]. In the quark sector, each 331-family can be assigned in 3 different ways. Therefore, in a phenomenological analysis, the allowed region associated with the $Z-Z^{\prime}$ mixing angle and the physical mass $M_{Z^{\prime}}$ of $Z^{\prime}$ will depend on the family assignment. We adopt the texture structure proposed in ref. [5] in order to obtain allowed regions for the $Z-Z^{\prime}$ mixing angle, the mass of the $Z^{\prime}$ boson and the values of $\beta$ for 3 different assignments of the quark families in mass eigenstates. The above analysis is performed through a $\chi^{2}$ statistics at $95 \%$ CL.

\section{THE QUARK AND NEUTRAL GAUGE SPECTRUM}

The fermion representations under $S U(3)_{c} \otimes S U(3)_{L} \otimes$ $U(1)_{X}$ read

$$
\begin{aligned}
& \widehat{\psi}_{L}=\left\{\begin{array}{l}
\widehat{q}_{L}:\left(\mathbf{3}, \mathbf{3}, X_{q}^{L}\right)=\left(\mathbf{3}, \mathbf{2}, X_{q}^{L}\right) \oplus\left(\mathbf{3}, \mathbf{1}, X_{q}^{L}\right), \\
\widehat{\ell}_{L}:\left(\mathbf{1}, \mathbf{3}, X_{\ell}^{L}\right)=\left(\mathbf{1}, \mathbf{2}, X_{\ell}^{L}\right) \oplus\left(\mathbf{1}, \mathbf{1}, X_{\ell}^{L}\right),
\end{array}\right. \\
& \widehat{\psi}_{L}^{*}=\left\{\begin{array}{l}
\widehat{q}_{L}^{*}:\left(\mathbf{3}, \mathbf{3}^{*},-X_{q}^{L}\right)=\left(\mathbf{3}, \mathbf{2}^{*},-X_{q}^{L}\right) \oplus\left(\mathbf{3}, \mathbf{1},-X_{q}^{L}\right), \\
\widehat{\ell}_{L}^{*}:\left(\mathbf{1}, \mathbf{3}^{*},-X_{\ell}^{L}\right)=\left(\mathbf{1}, \mathbf{2}^{*},-X_{\ell}^{L}\right) \oplus\left(\mathbf{1}, \mathbf{1},-X_{\ell}^{L}\right),
\end{array}\right. \\
& \widehat{\psi}_{R}=\left\{\begin{array}{l}
\widehat{q}_{R}:\left(\mathbf{3}, \mathbf{1}, X_{q}^{R}\right), \\
\widehat{\ell}_{R}:\left(\mathbf{1}, \mathbf{1}, X_{\ell}^{R}\right) .
\end{array}\right.
\end{aligned}
$$

The second equality comes from the branching rules $S U(2)_{L} \subset S U(3)_{L}$. The $X_{p}$ refers to the quantum number associated with $U(1)_{X}$. The generator of $U(1)_{X}$ conmute with the matrices of $S U(3)_{L}$; hence, it should take the form $X_{p} \mathbf{I}_{3 \times 3}$, the value of $X_{p}$ is related with the representations of $S U(3)_{L}$ and the anomalies cancellation. On the other hand, this fermionic content shows that the left-handed multiplets lie in either the 3 or $\mathbf{3}^{*}$ representations. In the framework of three family model, we recognize 3 different possibilities to assign the physical quarks in each family representation as shown in Table I in weak eigenstates.

On the other hand, we obtain the following mass eigenstates associated to the neutral gauge spectrum

$$
Z_{1 \mu}=Z_{\mu} C_{\theta}+Z_{\mu}^{\prime} S_{\theta} ; \quad Z_{2 \mu}=-Z_{\mu} S_{\theta}+Z_{\mu}^{\prime} C_{\theta}
$$

where a small mixing angle $\theta$ between the neutral currents $Z_{\mu}$ and $Z_{\mu}^{\prime}$ appears, with

$$
\begin{aligned}
& Z_{\mu}=C_{W} W_{\mu}^{3}-S_{W}\left(\beta T_{W} W_{\mu}^{8}+\sqrt{1-\beta^{2} T_{W}^{2}} B_{\mu}\right) ; \\
& Z_{\mu}^{\prime}=-\sqrt{1-\beta^{2} T_{W}^{2}} W_{\mu}^{8}+\beta T_{W} B_{\mu},
\end{aligned}
$$

where the Weinberg angle is defined as $S_{W}=$ $g^{\prime} /\left(\sqrt{g^{2}+\left(1+\beta^{2}\right) g^{\prime 2}}\right)$ and $g, g^{\prime}$ correspond to the coupling constants of the groups $S U(3)_{L}$ and $U(1)_{X}$, respectively.

\section{THE NEUTRAL GAUGE COUPLINGS}

The neutral Lagrangian associated to the SM-boson $Z_{1 \mu}$ in the weak basis of the SM quarks $U^{0}=\left(u^{0}, c^{0}, t^{0}\right)^{T}$ and $D^{0}=$ $\left(d^{0}, s^{0}, b^{0}\right)^{T}$, is

$$
\begin{aligned}
\mathcal{L}^{N C}= & \frac{g}{2 C_{W}}\left\{\overline{U^{0}} \gamma_{\mu}\left[G_{v}^{U(r)}-G_{a}^{U(r)} \gamma_{5}\right] U^{0}\right. \\
& \left.+\overline{D^{0}} \gamma_{\mu}\left[G_{v}^{D(r)}-G_{a}^{D(r)} \gamma_{5}\right] D^{0} Z_{1}^{\mu}\right\} .
\end{aligned}
$$

The couplings of the $Z_{1 \mu}$ have the same form as the SM couplings, where the usual vector and axial vector couplings $g_{V, A}^{S M}$ are replaced by $G_{V, A}^{(r)}=g_{V, A}^{S M} I+\delta g_{V, A}^{(r)}$, where 


\begin{tabular}{c|c|c}
\hline \hline Representation $A$ & Representation $B$ & Representation $C$ \\
\hline \hline$q_{m L}=\left(\begin{array}{c}d, s \\
-u,-c \\
J_{1}, J_{2}\end{array}\right)_{L}: \mathbf{3}^{*}$ & $q_{m L}=\left(\begin{array}{c}d, b \\
-u,-t \\
J_{1}, J_{3}\end{array}\right)_{L}: \mathbf{3}^{*}$ & $q_{m L}=\left(\begin{array}{c}s, b \\
-c,-t \\
J_{2}, J_{3}\end{array}\right)_{L}: \mathbf{3}^{*}$ \\
$q_{3 L}=\left(\begin{array}{c}t \\
b \\
J_{3}\end{array}\right)_{L}: \mathbf{3}$ & $q_{3 L}=\left(\begin{array}{c}c \\
s \\
J_{2}\end{array}\right)_{L}: \mathbf{3}$ & $q_{3 L}=\left(\begin{array}{c}u \\
d \\
J_{1}\end{array}\right)_{L}: \mathbf{3}$ \\
\hline
\end{tabular}

TABLE I: Three different family structures in the fermionic spectrum

$$
\delta g_{V, A}^{(r)}=\widetilde{g}_{V, A}^{(r)} S_{\theta},
$$

which corresponds to the correction due to the small $Z_{\mu}$ $Z_{\mu}^{\prime}$ mixing angle $\theta, \widetilde{g}_{V, A}^{(r)}$ the $Z_{\mu}^{\prime}$ coupling constants, and $r=$ $(A, B, C)$ each representation from Table I. The $Z_{\mu}^{\prime}$ couplings for leptons are

$$
\stackrel{\sim}{g}_{v, a}^{\ell}=\frac{g^{\prime} C_{W}}{2 g T_{W}}\left[\frac{-1}{\sqrt{3}}-\beta T_{W}^{2} \pm 2 Q_{\ell} \beta T_{W}^{2}\right],
$$

while for the quark couplings we get

$$
\stackrel{\sim}{g}_{v, a}^{q(r)}=\frac{g^{\prime} C_{W}}{2 g T_{W}} K^{(r) \dagger}\left(M \pm 2 Q_{q} \beta T_{W}^{2}\right) K^{(r)}
$$

with $q=U^{0}, D^{0}, \quad M=1 / \sqrt{3} \operatorname{diag}\left[1+\beta T_{W}^{2} / \sqrt{3}, 1+\right.$ $\left.\beta T_{W}^{2} / \sqrt{3},-1+\beta T_{W}^{2} / \sqrt{3}\right]$ and where we define for each representation from table I

$$
K^{(A)}=I, K^{(B)}=\left(\begin{array}{lll}
1 & 0 & 0 \\
0 & 0 & 1 \\
0 & 1 & 0
\end{array}\right), K^{(C)}=\left(\begin{array}{lll}
0 & 1 & 0 \\
0 & 0 & 1 \\
1 & 0 & 0
\end{array}\right) .
$$

We will consider linear combinations among the three families to obtain couplings in mass eigenstates by adopting an ansatz on the texture of the quark mass matrix in agreement with the CKM matrix. We take the structure of mass matrix suggested in ref. [5] given by $M_{q}^{\prime}=P_{q}^{\dagger} M P_{q}^{\dagger}$, with $P_{q}=\operatorname{diag}\left(\exp \left(\alpha_{1}\right), \exp \left(\alpha_{2}\right), \exp \left(\alpha_{3}\right)\right)$, and where $M$ is written in the basis $\left(u^{0}, c^{0}, t^{0}\right)$ or $\left(d^{0}, s^{0}, b^{0}\right)$ as

$$
M_{q}=\left(\begin{array}{ccc}
0 & A_{q} & A_{q} \\
A_{q} & B_{q} & C_{q} \\
A_{q} & C_{q} & B_{q}
\end{array}\right)
$$

For up-type quarks, $A_{U}=\sqrt{\frac{m_{t} m_{u}}{2}}, B_{U}=\left(m_{t}+m_{c}-m_{u}\right) / 2$ and $C_{U}=\left(m_{t}-m_{c}-m_{u}\right) / 2$; for down-type quarks $A_{D}=$ $\sqrt{\frac{m_{d} m_{s}}{2}}, B_{D}=\left(m_{b}+m_{s}-m_{d}\right) / 2$ and $C_{D}=-\left(m_{b}-m_{s}+\right.$ $\left.m_{d}\right) / 2$. The above ansatz is diagonalized by [5]

$$
R_{D}=\left(\begin{array}{ccc}
c & s & 0 \\
-\frac{s}{\sqrt{2}} & \frac{c}{\sqrt{2}} & -\frac{1}{\sqrt{2}} \\
-\frac{s}{\sqrt{2}} & \frac{c}{\sqrt{2}} & \frac{1}{\sqrt{2}}
\end{array}\right) ; R_{U}=\left(\begin{array}{ccc}
c^{\prime} & 0 & s^{\prime} \\
-\frac{s^{\prime}}{\sqrt{2}} & -\frac{1}{\sqrt{2}} & \frac{c^{\prime}}{\sqrt{2}} \\
-\frac{s^{\prime}}{\sqrt{2}} & \frac{1}{\sqrt{2}} & \frac{c^{\prime}}{\sqrt{2}}
\end{array}\right)
$$

with $c=\sqrt{m_{s} /\left(m_{d}+m_{s}\right)}, s=\sqrt{m_{d} /\left(m_{d}+m_{s}\right)}, c^{\prime}=$ $\sqrt{m_{t} /\left(m_{t}+m_{u}\right)}$ and $s^{\prime}=\sqrt{m_{u} /\left(m_{t}+m_{u}\right)}$. The complex matrix $M_{q}^{\prime}=P_{q}^{\dagger} M P_{q}^{\dagger}$ is then diagonalized by the bi-unitary transformation $U_{L q}^{\dagger} M_{q}^{\prime} U_{R q}$ with $U_{L q}=P_{q}^{\dagger} R_{q}$ and $U_{R q}=P_{q} R_{q}$. Then, the diagonal couplings in Eq. (5) in mass eigenstates have terms of the form $U_{L, R q}^{\dagger} G_{v, a}^{q(r)} U_{L, R q}=R_{q}^{\dagger} G_{v, a}^{q(r)} R_{q}$ where any effect of the $\mathrm{CP}$ violating phases $P$ disappears. Thus, we can write the Eq. (5) in mass eigenstates as

$$
\begin{aligned}
\mathcal{L}^{N C}= & \frac{g}{2 C_{W}}\left[\bar{U} \gamma_{\mu}\left(\mathfrak{G}_{v}^{U(r)}-\mathfrak{G}_{a}^{U(r)} \gamma_{5}\right) U\right. \\
& \left.+\bar{D} \gamma_{\mu}\left(\mathfrak{G}_{v}^{D(r)}-\mathfrak{G}_{a}^{D(r)} \gamma_{5}\right) D\right] Z_{1}^{\mu},
\end{aligned}
$$

where the couplings of quarks depend on the rotation matrix, with

$$
\mathfrak{G}_{v, a}^{q(r)}=g_{v, a}^{q} I+R_{q}^{\dagger} \delta g_{v, a}^{q(r)} R_{q}=g_{v, a}^{q} I+\delta \mathfrak{g}_{v, a}^{q(r)}
$$

We obtain flavor changing couplings in the quark sector due to the family dependence shown by $\widetilde{g}_{v, a}^{q(r)}$. All the analytical parameters $(O)$ at the $\mathrm{Z}$ pole have the same SM-form $\left(O_{S M}\right)$ but with small correction factors $(\delta O)$ which are expressed in terms of the coupling corrections $\delta \mathfrak{g}_{v, a}^{q(r)}$ that depend on the family assignment from table I. Each observable predicted by the 331 model takes the form $O_{331}=O_{S M}(1+\delta O)$. For the analysis, we take into account the observables at the $\mathrm{Z}$ pole shown in Table II from ref. [7], including data from atomic parity violation. The 331 corrections are 


$$
\begin{aligned}
\delta_{Z}= & \frac{\Gamma_{u}^{S M}}{\Gamma_{Z}^{S M}}\left(\delta_{u}+\delta_{c}\right)+\frac{\Gamma_{d}^{S M}}{\Gamma_{Z}^{S M}}\left(\delta_{d}+\delta_{s}\right) \\
& +\frac{\Gamma_{b}^{S M}}{\Gamma_{Z}^{S M}} \delta_{b}+3 \frac{\Gamma_{v}^{S M}}{\Gamma_{Z}^{S M}} \delta_{v}+3 \frac{\Gamma_{e}^{S M}}{\Gamma_{Z}^{S M}} \delta_{\ell} \\
\delta_{h a d}= & R_{c}^{S M}\left(\delta_{u}+\delta_{c}\right)+R_{b}^{S M} \delta_{b}+\frac{\Gamma_{d}^{S M}}{\Gamma_{h a d}^{S M}}\left(\delta_{d}+\delta_{s}\right) ; \\
\delta_{\sigma}= & \delta_{h a d}+\delta_{\ell}-2 \delta_{Z} \\
\delta A_{f}= & \frac{\delta_{V}^{f f}}{g_{V}^{f}}+\frac{\delta_{A}^{f f}}{g_{A}^{f}}-\delta_{f}, \\
\delta Q_{W}= & \frac{\Delta Q_{W}}{Q_{W}^{S M}}
\end{aligned}
$$

where

$$
\begin{gathered}
\delta_{f}=\frac{2 g_{v}^{f} \delta \mathfrak{g}_{v}^{f f}+2 g_{a}^{f} \delta \mathfrak{g}_{a}^{f f}}{\left(g_{v}^{f}\right)^{2}+\left(g_{a}^{f}\right)^{2}}, \\
\Delta Q_{W} \simeq-0.01+\Delta Q_{W}^{\prime}, \\
\Delta Q_{W} \simeq-0.01-16(2 Z+N)\left[\left(g_{A}^{e} \mathfrak{g}_{v}^{u u}+\tilde{g}_{a}^{e} g_{V}^{u}\right)\right. \\
\left.+(Z+2 N)\left(g_{a}^{e} \mathfrak{g}_{v}^{d d}+\tilde{g}_{a}^{e} g_{v}^{d}\right)\right] S_{\theta} \\
\left.-16\left[(2 Z+N) \tilde{g}_{a}^{e} \mathfrak{g}_{v} u(Z+2 N) \tilde{g}_{a}^{e} \mathfrak{g}_{v}\right] d\right] \frac{M_{Z_{Z^{\prime}}}^{2}}{M_{Z_{2}}^{2}}(16)
\end{gathered}
$$

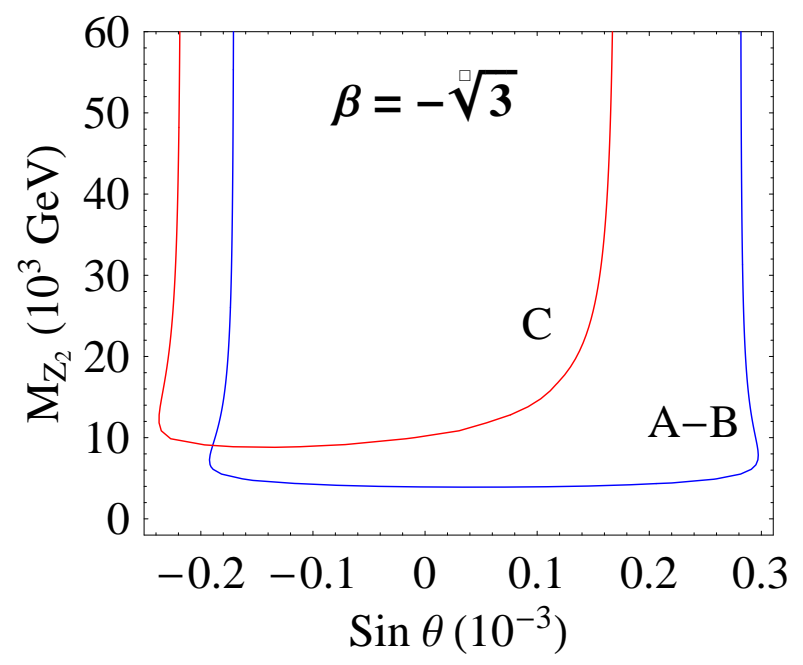

FIG. 1: Allowed region for 331 models with $\beta=-\sqrt{3}$ in the $M_{Z_{2}}-$ $S_{\theta}$ plane. The regions are dispayed for $A, B$ and $C$ representation.

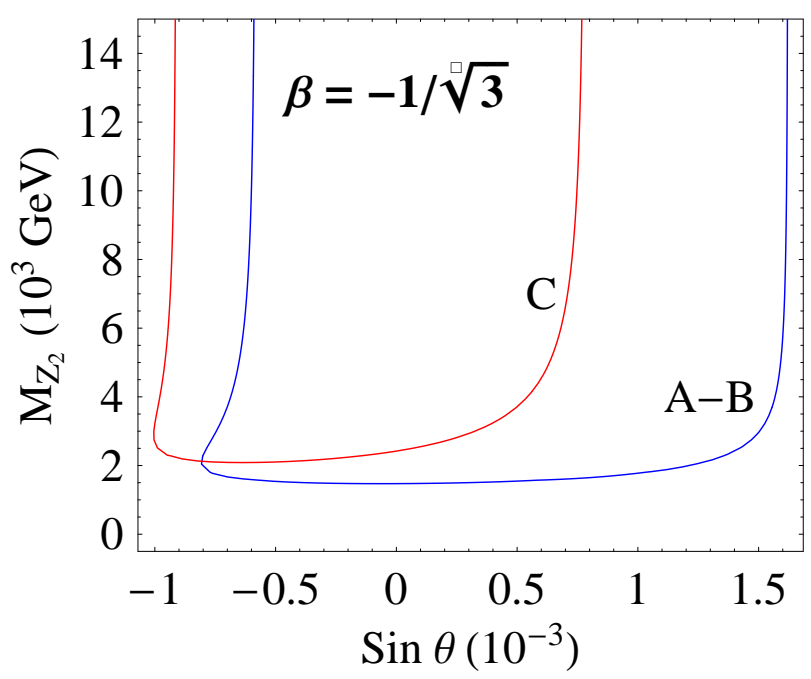

FIG. 2: Allowed region for 331 models with $\beta=-1 / \sqrt{3}$ in the $M_{Z_{2}}-S_{\theta}$ plane. The regions are dispayed for $A, B$ and $C$ representation.

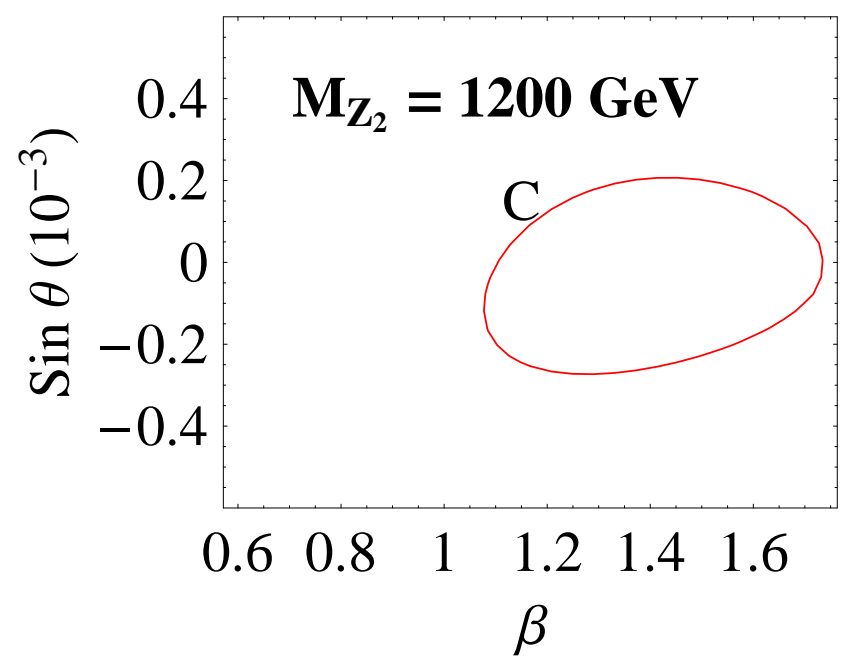

FIG. 3: Allowed region for $M_{Z_{2}}=1200 \mathrm{GeV}$ in the $S_{\theta}-\beta$ plane. Only the $C$ representation exhibit allowed region.

\section{PRECISION FIT TO THE Z-POLE OBSERVABLES}

With the expressions for the Z-pole observables and the experimental data from the LEP [7], we perform a $\chi^{2}$ fit for each representation $A, B$ and $C$ at $95 \% \mathrm{CL}$ and 3 d.o.f, where the free quantities $S_{\theta}, M_{Z_{2}}$ and $\beta$ can be constrained at the $Z_{1}$ peak. Figs. 1 and 2 show the allowed region for the main versions of 331 models corresponding to $\beta=-\sqrt{3}$ [2] and $\beta=-\frac{1}{\sqrt{3}}$ [3], respectively, which exhibits family-dependent regions. First of all, we note that $A$ and $B$ representations display broader mixing angles than representation $C$. For the model $\beta=-\sqrt{3}$, we see that the lowest bound in the $M_{Z_{2}}$ value is about $4000 \mathrm{GeV}$ for $A$ and $B$ cases, while for the $C$ representation this bound increses to $10000 \mathrm{GeV}$, showing an strong dependence on the family representation. The model 


\begin{tabular}{c|c|c|c}
\hline Quantity & Experimental Values & Standard Model & 331 Model \\
\hline \hline$\Gamma_{Z}[\mathrm{GeV}]$ & $2.4952 \pm 0.0023$ & $2.4972 \pm 0.0012$ & $\Gamma_{Z}^{S M}\left(1+\delta_{Z}\right)$ \\
\hline$\Gamma_{\text {had }}[\mathrm{GeV}]$ & $1.7444 \pm 0.0020$ & $1.7435 \pm 0.0011$ & $\Gamma_{h a d}^{S M}\left(1+\delta_{h a d}\right)$ \\
\hline$\Gamma_{\left(\ell^{+} \ell^{-}\right)} \mathrm{MeV}$ & $83.984 \pm 0.086$ & $84.024 \pm 0.025$ & $\Gamma_{\left(\ell^{+} \ell^{-}\right)}^{S M}\left(1+\delta_{\ell}\right)$ \\
\hline$\sigma_{h a d}[n b]$ & $41.541 \pm 0.037$ & $41.472 \pm 0.009$ & $\sigma_{h a d}^{S M}\left(1+\delta_{\sigma}\right)$ \\
\hline$R_{e}$ & $20.804 \pm 0.050$ & $20.750 \pm 0.012$ & $R_{e}^{S M}\left(1+\delta_{h a d}+\delta_{e}\right)$ \\
\hline$R_{\mu}$ & $20.785 \pm 0.033$ & $20.751 \pm 0.012$ & $R_{\mu}^{S M}\left(1+\delta_{\text {had }}+\delta_{\mu}\right)$ \\
\hline$R_{\tau}$ & $20.764 \pm 0.045$ & $20.790 \pm 0.018$ & $R_{\tau}^{S M}\left(1+\delta_{h a d}+\delta_{\tau}\right)$ \\
\hline$R_{b}$ & $0.21638 \pm 0.00066$ & $0.21564 \pm 0.00014$ & $R_{b}^{S M}\left(1+\delta_{b}-\delta_{h a d}\right)$ \\
\hline$R_{c}$ & $0.1720 \pm 0.0030$ & $0.17233 \pm 0.00005$ & $R_{c}^{S M}\left(1+\delta_{c}-\delta_{h a d}\right)$ \\
\hline$A_{e}$ & $0.15138 \pm 0.00216$ & $0.1472 \pm 0.0011$ & $A_{e}^{S M}\left(1+\delta A_{e}\right)$ \\
\hline$A_{\mu}$ & $0.142 \pm 0.015$ & $0.1472 \pm 0.0011$ & $A_{\mu}^{S M}\left(1+\delta A_{\mu}\right)$ \\
\hline$A_{\tau}$ & $0.136 \pm 0.015$ & $0.1472 \pm 0.0011$ & $A_{\tau}^{S M}\left(1+\delta A_{\tau}\right)$ \\
\hline$A_{b}$ & $0.925 \pm 0.020$ & $0.9347 \pm 0.0001$ & $A_{b}^{S M}\left(1+\delta A_{b}\right)$ \\
\hline$A_{c}$ & $0.670 \pm 0.026$ & $0.6678 \pm 0.0005$ & $A_{c}^{S M}\left(1+\delta A_{c}\right)$ \\
\hline$A_{s}$ & $0.895 \pm 0.091$ & $0.9357 \pm 0.0001$ & $A_{s}^{S M}\left(1+\delta A_{s}\right)$ \\
\hline$A_{F B}^{(0, e)}$ & $0.0145 \pm 0.0025$ & $0.01626 \pm 0.00025$ & $A_{F B}^{(0, e) S M}\left(1+2 \delta A_{e}\right)$ \\
\hline$A_{F B}^{(0, \mu)}$ & $0.0169 \pm 0.0013$ & $0.01626 \pm 0.00025$ & $A_{F B}^{(0, \mu) S M}\left(1+\delta A_{e}+\delta A_{\mu}\right)$ \\
\hline$A_{F B}^{(0, \tau)}$ & $0.0188 \pm 0.0017$ & $0.01626 \pm 0.00025$ & $A_{F B}^{(0, \tau) S M}\left(1+\delta A_{e}+\delta A_{\tau}\right)$ \\
\hline$A_{F B}^{(0, b)}$ & $0.0997 \pm 0.0016$ & $0.1032 \pm 0.0008$ & $A_{F B}^{(0, b) S M}\left(1+\delta A_{e}+\delta A_{b}\right)$ \\
\hline$A_{F B}^{(0, c)}$ & $0.0706 \pm 0.0035$ & $0.0738 \pm 0.0006$ & $A_{F B}^{(0, c) S M}\left(1+\delta A_{e}+\delta A_{c}\right)$ \\
\hline$A_{F B}^{(0, s)}$ & $0.0976 \pm 0.0114$ & $0.1033 \pm 0.0008$ & $A_{F B}^{(0, s) S M}\left(1+\delta A_{e}+\delta A_{s}\right)$ \\
\hline$Q_{W}(C s)$ & $-72.69 \pm 0.48$ & $-73.19 \pm 0.03$ & $Q_{W}^{S M}\left(1+\delta Q_{W}\right)$ \\
\hline & & &
\end{tabular}

TABLE II: The Z-pole parameters for experimental values, SM predictions and 331 corrections.

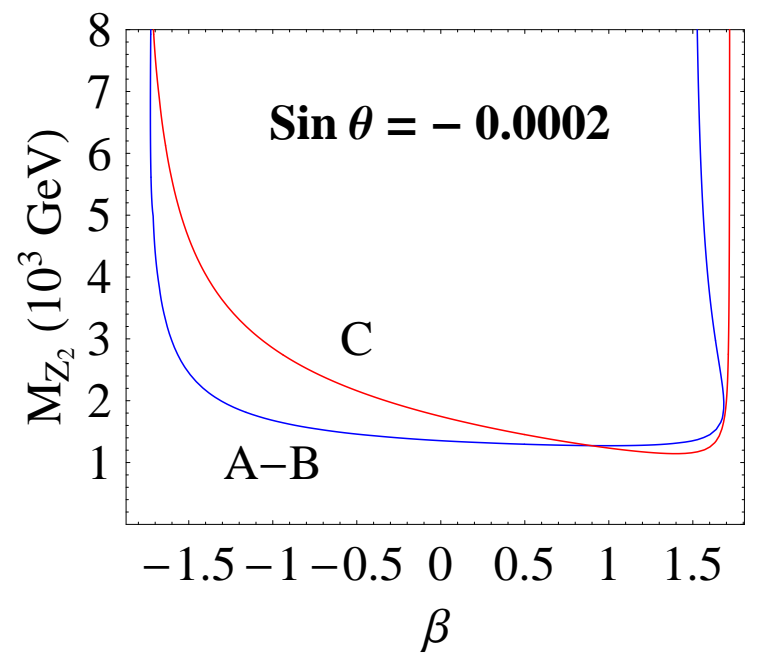

FIG. 4: Allowed region for $S_{\theta}=-0.0002$ in the $M_{Z_{2}}-\beta$ plane. The regions are dispayed for $A, B$ and $C$ representations.

$\beta=-\frac{1}{\sqrt{3}}$ exhibits a lower bound in the $Z_{2}$ mass, where the lowest bound is about $1400 \mathrm{GeV}$ for $A$ and $B$ regions, and $2100 \mathrm{GeV}$ for the $C$ spectrum. We also see that the mixing angle in this model is smaller by about one order of magnitude than the angles predicted by the $\beta=-\sqrt{3}$ model.

On the other hand, we get the best allowed region in the plane $S_{\theta}-\beta$ for two different values of $M_{Z_{2}}$. The lowest bound that display an allowed region is about $1200 \mathrm{GeV}$, which ap- pears only for the $C$ assignments such as Fig. 3 shows. We can see in this case that the usual 331 models are excluded, and only those 331 models with $1.1 \lesssim \beta \lesssim 1.75$ are allowed with small mixing angles $\left(S_{\theta} \sim 10^{-4}\right)$. Fig. 4 display the allowed region in the $M_{Z_{2}}-\beta$ plane for a small mixing angle ( $\left.S_{\theta}=-0.0002\right)$. It is noted that the smallest bounds in $M_{Z_{2}}$ is obtained for $\beta>0$. 
[1] S. Godfrey arXiv: hep-ph/0201093; G. Weiglein et. al., arXiv: hep-ph/0410364; R.D. Heuer et. al, arXiv: hep-ph/0106315.

[2] F. Pisano and V. Pleitez, Phys. Rev. D 46, 410 (1992); P.H. Frampton, Phys. Rev. Lett. 69, 2889 (1992).

[3] R. Foot, H.N. Long, and T.A. Tran, Phys. Rev. D 50, R34 (1994).

[4] L.A. Sánchez, W.A. Ponce, and R. Martínez, Phys. Rev. D 64, 075013 (2001);Rodolfo A. Diaz, R. Martinez, and F. Ochoa, Phys. Rev. D 69, 095009 (2004); Rodolfo A. Diaz, R. Martinez, and F. Ochoa, Phys. Rev. D 72, 035018 (2005).

[5] K. Matsuda, H. Nishiura, Phys. Rev. D 69, 053005 (2004).

[6] Fredy Ochoa and R. Martinez, Phys. Rev. D 72, 035010 (2005).

[7] S. Eidelman et. al. Particle Data Group, Phys. Lett. B 592, 120 (2004); S. Schael et. al. arXiv: hep-ph/0509008 (submitted to Physics Reports) 